\title{
End-Stage Renal Disease and Lower Gastrointestinal Bleeding - A Propensity-matched Analysis of Nationwide Inpatient Sample.
}

\author{
Pavani Reddy Garlapati ${ }^{1}$, Bhavesh Gajjar ${ }^{2}$, Eric Then $^{1}$, and Vijay Gayam ${ }^{3}$ \\ ${ }^{1}$ Affiliation not available \\ ${ }^{2}$ East Tennessee State University \\ ${ }^{3}$ Interfaith Medical Center
}

June 4, 2020

\begin{abstract}
Introduction: We aim to determine the influence of lower gastrointestinal bleeding (LGIB) on mortality, morbidity, length of hospital stay, and resource utilization in end-stage renal disease (ESRD) patients. Material and Methods: The National Inpatient Sample database (2016 \&2017) was used for data analysis using the International Classification of Diseases, Tenth Revision codes to identify the patients with the principal diagnosis of ESRD and LGIB. We assessed the all-cause in-hospital mortality, morbidity, predictors of mortality, length of hospital stay (LOS), and total costs between propensity-matched groups of ESRD patients with LGIB versus ESRD patients. Results: We identified 2187954 ESRD patients, of whom 242075 has LGIB, and 1945879 were ESRD patients. The in-hospital mortality was higher in ESRD with LGIB (OR 2.5, 95\% CI 1.5-2.2; P=0.00). ESRD with LGIB has higher odds of mechanical ventilation (OR 1.4, 95\% CI 6.4-16.4; $\mathrm{P}=0.00$ ), and shock requiring vasopressor (OR 1.2, 95\% CI 4.9-5.4; $\mathrm{P}=0.002$ ). Advanced age (OR 1.02 CI 1.02-1.03 $\mathrm{P}=0.00$ ), anemia (OR 1.04 CI 1.59-1.91 P=0.006), acute coronary syndrome (OR $1.8 \mathrm{CI} 1.6-2.1, \mathrm{P}=0.00$ ), acute respiratory failure (OR 1.29 CI 2.0-2.6, $\mathrm{P}=0.00$ ), mechanical ventilation ( $\mathrm{OR} 1.9$, CI 3.5-4.4, $\mathrm{P}=0.00$ ), and sepsis ( $\mathrm{OR} 1.5$, CI 4.1-5.08, $\mathrm{P}=0.00$ ) were identified as predictors of mortality in ESRD with LGIB. Mean LOS $(10.8 \pm 14.9$ vs. $6.3 \pm 8.5, \mathrm{P}<0.01)$ and mean total charges $(37054 \$$ vs. $18080 \$, \mathrm{P}<0.01)$ were also higher. Conclusions: In this propensity-matched analysis, ESRD with LGIB was associated with higher odds of in-hospital mortality, mechanical ventilation, and shock requiring vasopressor. Mean LOS and resource utilization were also higher.
\end{abstract}

\section{Introduction:}

Gastrointestinal bleeding (GIB) is a considerable discharge diagnosis with > 500,000 discharges annually. GIB has approximately 5 billion dollars cost for hospitalization. In the last two decades, research and advancement have improved inpatient mortality of upper GIB. While the rate of lower GIB and in-hospital complication rate has gone up, it is creating an economic burden for hospitals. [1-4] Patients with chronic kidney disease (CKD) and end-stage renal disease (ESRD) have a higher tendency for gastrointestinal bleeding (GIB). [5 6] These patients have a five-fold higher risk of GIB as compared with those without CKD. [7-9] Also, patients with CKD have higher mortality [10]. ESRD patients are likely to bleed more due to a variety of causes, which include heparin used during dialysis, platelet dysfunction, and medications $\left[\begin{array}{lll}7 & 11 & 12\end{array}\right]$.

Multiple studies have shown unfavorable outcomes in ESRD patients who develop upper GIB. Not many studies have examined the impact of ESRD on patients with lower gastrointestinal bleeding (LGIB) [5 1315]. We aimed to study ESRD patients who are hospitalized for acute LGIB. We have assessed mortality, morbidity, and predictors for mortality of these patient groups. 


\section{Methods:}

\section{Study Data:}

In this retrospective analysis, we obtained data from the 2016 and 2017 National Inpatient Sample (NIS), which is sponsored by the Agency for Healthcare Research and Quality as a part of the Healthcare Cost and Utilization Project (HCUP). NIS is the largest publicly available all-payer administrative database, containing data on more than 7 million hospitalizations (unweighted); when weighted, it represents about 35 million hospitalizations nationally. It provides information on clinical and resource utilization with safeguards to protect data for individual patients, physicians, and hospitals. Beginning in October 2015, the NIS started using the International Classification of Diseases, Tenth Edition, Clinical Modification/Procedure Coding System (ICD-10-CM/PCS) to reflect the implementation of ICD-10-CM/PCS by hospital systems. Using the Agency for Healthcare Research and Quality sampling and weighting method, national estimates of the entire U.S. hospitalized population were calculated. [16]

\section{Study design:}

Given the de-identified nature of the NIS data, our study was exempt from approval from the Institutional Review Board. We identified all patients ([?]18 years of age) who had a discharge diagnosis of ESRD (n $=2187954$ ), using their respective ICD-10-CM/PCS codes. We divided the total sample into two groups: ESRD with LGIB $(n=242075)$ and ESRD only $(n=1945879)$. We identified patients with LGIB using appropriate diagnosis codes. The ICD-10-CM/PCS codes used in this study are displayed in supplementary tables 1 and 2 .

For baseline characteristics, we used patient demographics (age, race, and sex), the Charlson Comorbidity Index, insurance status, hospital characteristics, and relevant comorbidities coronary artery disease (CAD) or CAD equivalent, hypertension (HTN), obesity, dyslipidemia, diabetes mellitus (D.M.), chronic lung disease, tobacco smoking, alcohol use, peripheral vascular disease, blood thinner use (anticoagulants/antithrombotics/antiplatelets) and congestive heart failure (CHF) (Table 1). Comorbidities were identified using their respective ICD-10-CM/PCS codes (supplementary table 1)).

\section{Outcomes:}

The primary outcome of interest was all-cause in-hospital mortality and predictors of mortality. The secondary outcomes included the incidence of sepsis, acute coronary syndrome, shock requiring vasopressors, acute respiratory failure, disseminated intravascular coagulation, and mechanical ventilation. Complications were identified using their respective ICD-10-CM/PCS (supplementary tables 1 and 2). We also studied the length of hospital stay (LOS) and average hospital costs.

\section{Statistical Analysis:}

We conducted all statistical analyses as per the recommended methods accounting for the intricate survey design of the NIS database. [17] Categorical data are reported as frequency and percentage, and continuous data as mean with standard deviation and standard error. Categorical variables were analyzed using Pearson's Chi-square test, and continuous variables were analyzed using the Student's t-test. Unadjusted odds ratios for the primary and secondary outcomes were calculated using univariate logistic regression. Multivariable logistic regression was used to adjust for potential confounders in the final model. Statistical significance was set at a two-sided p-value of $<0.05$. STATA/ MP 15.10 (Stata Corp LLC) was used for statistical analysis. All analyses in our study were weighted using provided discharge weights to produce national estimates. Hospital costs were inflation-adjusted for 2018 using the Consumer Price Index (provided by the U.S. Department of Labor). 
To account for the differences in baseline characteristics, we used propensity score matching. [18 19] To establish a propensity-matched cohort, we used the treatment outcomes as the outcome variable and potential confounders as covariates. A 1:1 propensity score-match was performed using a caliper width of 0.1 using the psmatch 2 command. Appropriate Caliper was calculated by multiplying 0.2 with a standard deviation of the logit of the propensity score. Pstest was used to generate the unmatched and matched variable. The standardized difference of $<10 \%$ checked with pbalchk command suggested adequacy of the match between two groups among the measured covariates. Regression analysis was done using a generalized linear model using all covariates in succession in the final model, including patient-level discharge weights. We added covariates one by one in the model, and if the coefficient changed by more than $20 \%$, we included that covariate in the final model. A full list of covariates used in the regression analysis and confounders in the multivariable regression model is shown in the supplemental table 2.

\section{Results:}

\section{Characteristics of the Study Population:}

In 2016 and 2017, we identified 2187954 patients admitted with the diagnosis of ESRD. Of these, 242075 are ESRD with LGIB, and 1945879 ESRD only. The mean age in the ESRD with LGIB and ESRD groups was $64.4 \pm 14.2$ years and $61.4 \pm 15.6$ years, respectively. Before propensity matching, there was a statistically significant difference observed with age, sex, race, hospital bed size, hospital region, discharge disposition, hospital teaching status, insurance, Charlson comorbidity index. There was also a significant difference with the comorbidities, which included diabetes (D.M.), obesity, dyslipidemia, coronary artery disease (CAD), chronic lung disease and CHF [Table 1].

All covariates from were used to generate a propensity score. We assessed the matched cohorts for covariate balance. As shown in , matching eliminated almost all significant differences in clinical characteristics, demographics, hospital characteristics, payment source, and comorbidity prevalence between the two cohorts.

\section{Comparing Primary and Secondary Outcomes:}

We compared the outcomes between ESRD with LGIB vs. ESRD groups. Table 3 shows the proportion of primary and secondary outcomes in both groups. In the unmatched cohort, there was a statistically significant difference observed with the in-hospital mortality (OR 1.3, 95\% CI 1.2-1.3; $\mathrm{P}=0.00$ ). After propensity score matching, the results are consistent as there was a statistically significant difference in the in-hospital mortality between the two groups (OR 2.5, 95\% CI 1.5-2.2; $\mathrm{P}=0.00$ ). (Table 3 )

In the unmatched cohort, there were higher odds of sepsis (OR 1.4, 95\% CI 1.4-1.5; P=0.00), acute respiratory failure (OR 1.6, 95\% CI 1.5-1.7; $\mathrm{P}=0.00$ ), mechanical ventilation ( $\mathrm{OR} 1.8,95 \%$ CI 1.8-1.9; $\mathrm{P}=0.00$ ), shock requiring vasopressor (OR 1.9, 95\% CI 1.8-2.1; $\mathrm{P}=0.00$ ), $\mathrm{DIC}$ (OR 2.9, 95\% CI 4.4-5.5; $\mathrm{P}=0.00$ ), and acute coronary syndrome (OR 1.6, 95\% CI 1.5-1.6; $\mathrm{P}=0.00$ ), in the ESRD with LGIB group compared to the ESRD group. After matching the cohorts, there were higher odds of mechanical ventilation (OR 1.4, 95\% CI 6.04-16.4; $\mathrm{P}=0.00$ ), shock requiring vasopressor, and blood transfusion (OR 9.6, 95\% CI 2.3-4.0; $\mathrm{P}=0.002$ ). There was no difference observed with the other complications, including sepsis, acute coronary syndrome, and acute respiratory failure. (Table 3)

\section{Predictors of Mortality in ESRD with LGIB}

Multivariate logistic regression was performed to identify the predictors of mortality in ESRD with LGIB group. Advanced age (OR 1.02 CI 1.02-1.03 P=0.00), anemia (OR 1.04 CI 1.59-1.91 $\mathrm{P}=0.006$ ), acute coronary syndrome (OR 1.8 CI 1.6-2.1, $\mathrm{P}=0.00$ ), acute respiratory failure (OR 1.29 CI 2.0-2.6, $\mathrm{P}=0.00$ ), mechanical ventilation (OR 1.9, CI 3.5-4.4, $\mathrm{P}=0.00$ ), and sepsis ( $\mathrm{OR} 1.5$, CI 4.1-5.08, $\mathrm{P}=0.00$ ) were identified as predictors of mortality. (Table 4)

\section{Length of Stay and Resource Utilization:}


There was statistical significance observed with the mean length of stay (LOS) and the mean cost of care between the two groups. Higher mean LOS $(10.8 \pm 14.9$ vs. $6.3 \pm 8.5, \mathrm{P}<0.01)$ and mean total charges $(37054$ $\$$ vs. $18080 \$, \mathrm{P}<0.01$ ) were observed in the ESRD with LGIB group. (Table 5)

\section{Discussion:}

The present study is the first and the largest to assess the outcomes of ESRD with LGIB. We analyzed 2187954 admissions from the NIS database to examine outcomes of ESRD among the patients with LGIB. To compare outcomes of ESRD among patients with LGIB versus ESRD patients, we used a propensitymatched model. This model allowed us to account for confounding factors and reduce the effect of selection bias. The in-hospital mortality was higher in ESRD patients with LGIB group. This group has higher odds of requiring mechanical ventilation and vasopressor for the shock. Advanced age, acute coronary syndrome, anemia, acute respiratory failure, mechanical ventilation, and sepsis were identified as predictors of mortality in this group. Mean LOS and mean total charges were also higher in this group.

ESRD is a growing public health problem with number tripled between 1990 and 2010. ESRD patients have a higher tendency to develop LGIB. As the primary etiological factors (diabetes and hypertension) for ESRD are increasing, there will be an increasing rate of ESRD, which might as well increase the case of GIB related to ESRD. [6 20] ESRD patients are likely to bleed more due to a variety of causes, which include heparin used during dialysis, platelet dysfunction, and medications. [ [ 711 12] It is essential to understand that LGIB in ESRD patients could be due to a variety of causes. [21] The most common cause of bleeding is angiodysplasia. The other causes are diverticulosis, hemorrhoids, stercoral ulcer, and ischemic colitis. Due to many pathophysiology and locations of bleeding, ESRD patients who develop acute LGIB require a thorough evaluation.

In 2016, there were 35.7 million hospital stays in the United States, and the cost of these stays totaled over $\$ 417$ billion, with a mean cost per stay of $\$ 11,700$ [22]. Hospitals and health systems across the nation are under pressure to avoid patient harm and to reduce the length of stay (LOS) [23]. A longer LOS also increases the likelihood of a hospital-acquired condition (HAC), which harms patients and contributes to an even costlier and longer stay [24 25]. The mean LOS is higher among ESRD patients with LGIB compared to ESRD patients. Our findings are consistent with previous studies which have shown that the patients with CKD have an average hospital length of stay (LOS) that is 2 to 3 days longer compared to the general population [26 27]. Increased LOS results in increased cost of a stay in the hospital. Our results show that the mean cost of hospitalization in ESRD patients who develop LGIB is $19000 \$$ more compared with ESRD patients. Early identification and intervention would be prudent to reduce the length and cost of the stay.

Many studies have shown increased mortality for ESRD patients with GIB. The all-cause mortality of ESRD patients hospitalized for UGIB is significantly high, with the first-month mortality of 11 to $14 \%$, and the one-year mortality could go up to 27\%. [28 29] There is limited data available for ESRD patients hospitalized for LGIB. A study by Trivedi et at; showed an episode of hospital-associated gastrointestinal bleeding in long-term dialysis patients could increase the hazard of death by two-fold [5]. Our findings show that ESRD patients who get hospitalized for LGIB are likely to have more mortality (OR 1.3, 95\% CI 1.2-1.3; $\mathrm{P}=0.00)$ compared with ESRD patients. ESRD patients have many comorbid conditions and higher mortality due to cardiovascular disease. Cardiovascular events may increase after gastrointestinal bleeding causing hemodynamic insult or decline in hemoglobin [5]. GIB would cause hypovolemia. Hypovolemia from any cause increases mortality in the patients hospitalized for GIB. [30] To reduce mortality in ESRD patients with LGIB, early volume resuscitation, and adequate blood transfusion might be necessary. Also, in the patient with a history of coronary artery disease, the optimization of risk factors and medicines is of very high value to reduce mortality.

ESRD patients who are hospitalized for LGIB have higher odds of requiring mechanical ventilation (OR 1.4, 95\% CI 6.4-16.4; $\mathrm{P}=0.00$ ) and vasopressor for shock (OR 1.2, 95\% CI 4.9-5.4; $\mathrm{P}=0.002$ ). These could be reasoned as these patients have many comorbidities, and they are likely to get sicker quickly. [30] We hypothesized that patients with ERSD and LGIB have a longer LOS and likely to develop more complications. 
Complications like cardio-respiratory failure and sepsis would require them to need more ventilator and vasopressor support. Early identification of shock, volume status, and sepsis would be prudent to improve outcomes in ESRD patients and LGIB.

In this study, we have identified a few predictors for increased mortality in ESRD patients who get hospitalized for LGIB. These predictors are advanced age (OR 1.02 CI 1.02-1.03 $\mathrm{P}=0.00$ ), anemia (OR 1.04 CI 1.59-1.91 $\mathrm{P}=0.006$ ), acute coronary syndrome (OR 1.8 CI 1.6-2.1, $\mathrm{P}=0.00$ ), acute respiratory failure (OR 1.29 CI 2.0-2.6, $\mathrm{P}=0.00$ ), mechanical ventilation (OR 1.9, CI 3.5-4.4, $\mathrm{P}=0.00$ ), and sepsis (OR 1.5, CI 4.1-5.08, $\mathrm{P}=0.00)$. ESRD patients have a high number of comorbid conditions. One study for LGIB has shown that in-hospital mortality increased $>7 \%$ with comorbid score [?] 2 vs. 1.7\% for those with no comorbidities [30]. We are going to emphasize the importance of recognizing that ESRD patients who get hospitalized for LGIB need close attention as they have a higher likelihood of becoming sicker and dying while hospitalized. These predictors could help in the future to create a risk score to identify high-risk patients earlier during hospitalization.

The primary limitation of the study is the cross-sectional design of the data, which does not allow longitudinal follow-up of patients, limiting us from incorporating a temporal effect into our analysis. Second, the NIS contains hospitalization records and not individual patients implying that adjusting for multiple hospitalizations was impossible. Despite these limitations, our study diminishes bias similarly to a randomized trial by including a wide array of covariates in both our propensity matching algorithm and our final models. We were able to provide the results of a large cohort composed of patients from numerous institutions, all geographic regions, economic and demographic groups in the USA. Therefore, our results could be generalized to the USA population.

In summary, ESRD patients who get hospitalized for LGIB are sicker than we believe. They have a higher chance of requiring critical care support leading to increased length of stay and cost for care. These patients have a higher tendency to bleed, and they bleed more due to a variety of insults, and due to a few different causes. Clinicians are compelled to have a broader knowledge of this clinical entity as the management of these patients involves a variety of tests and treatments. In the future, developing a risk score to identify the high-risk patients would be of help.

Table 1. Demographicscomparing ESRD with LGIB vs. ESRD only.

\begin{tabular}{llll}
\hline Variable & ESRD with LGIB (\%) & ESRD without LGIB (\%) & $p$-value \\
\hline Total & 242075 & 1945879 & \\
Age (Mean \pm S D) & $64.4 \pm 14.2$ & $61.4 \pm 15.6$ & $0.00^{*}$ \\
Female & 45.7 & 46.4 & \\
Race & & & \\
Caucasian (\%) & 41.27 & 44.02 & $0.00^{*}$ \\
African American (\%) & 33.9 & 33.2 & \\
Hispanic (\%) & 16.7 & 14.19 & \\
Asian (\%) & 3.8 & 4.6 & \\
Native American (\%) & 1.1 & 1.08 & $0.00^{*}$ \\
Others (\%) & 3.04 & 2.8 & \\
Hospital Bed size (\%) & & & \\
Small & 14.4 & 15.7 & $0.00^{*}$ \\
Medium & 27.5 & 29.3 & \\
Large & 58.02 & 54.8 & \\
Hospital region (\%) & & 16.8 & \\
Northeast & 17.1 & 19.8 & \\
Midwest & 21.6 & 43.3 & \\
South & 41.6 & 19.9 & \\
West & 19.5 & &
\end{tabular}




\begin{tabular}{llll}
\hline Variable & ESRD with LGIB (\%) & ESRD without LGIB (\%) & $p$-value \\
\hline Discharge & & & \\
Routine & 52.4 & 40.2 & $0.00^{*}$ \\
Skill Nursing Facility & 2.5 & 2.8 & \\
Charlson comorbidity Index (\%) & & & \\
0 or 1 & 81.5 & 79.0 & $0.01^{*}$ \\
2 & 10.4 & 11.6 & \\
3 & 7.9 & 9.2 & $0.00^{*}$ \\
Insurance Type (\%) & & & \\
Medicare (\%) & 77.1 & 72.8 & 0.50 \\
Medicaid (\%) & 9.2 & 13.0 & \\
Private (\%) & 10.8 & 10.9 & 0.1 \\
Uninsured (\%) & 1.1 & 0.01 & 0.19 \\
Teaching Hospital (\%) & 75.03 & 74.7 & 0.2 \\
Chronic Co-morbidity & & & 0.1 \\
DM (\%) & 59 & 61.4 & $0.002^{*}$ \\
HTN (\%) & 1.6 & 1.5 & 0.2 \\
Obesity (\%) & 15.1 & 15.9 & 0.8 \\
Dyslipidemia (\%) & 40.7 & 41.9 & 0.1 \\
CAD (\%) & 38.5 & 35.9 & 0.3 \\
CHF (\%) & 36.2 & 35.2 & 0.3 \\
PVD (\%) & 10.1 & 9.9 & $0.00^{*}$ \\
Tobacco Smoking (\%) & 23.2 & 21.1 & \\
Alcohol Use 9\%) & $12 . .5$ & 13.1 & 11.5 \\
Blood Thinners (\%) & 12.7 & 9.2 & \\
Chronic Lung disease (\%) & 11.5 & & \\
\hline
\end{tabular}

* denotes statistically significantly different result

DM, diabetes mellitus, HTN, hypertension, CAD, coronary artery disease, CHF, congestive heart failure, PVD, peripheral vascular disease

Table 2. Balance of covariates after propensity score matching.

\begin{tabular}{lllll}
\hline Covariates & ESRD with LGIB (\%) & ESRD with LGIB (\%) & ESRD without LGIB (\%) & $p$-value \\
\hline Mean Age & 64.4 & 64.4 & 64.1 & 0.12 \\
Female & 45.2 & 45.2 & 45.0 & 0.87 \\
Race & & & \\
Caucasian (\%) & 55.1 & 55.1 & 54.9 & \\
African American (\%) & 35.2 & 35.2 & 35.8 & 0.19 \\
Hispanic (\%) & 1.3 & 1.3 & 1.2 & \\
Asian (\%) & 4.6 & 4.6 & 4.5 & \\
Native American (\%) & 1.0 & 1.0 & 1.2 & \\
Others (\%) & 2.8 & 2.8 & 2.4 & \\
Hospital Bed size (\%) & & & \\
Small & 14.5 & 14.5 & 15.5 & \\
Medium & 27.5 & 27.5 & 26.9 & \\
Large & 58.0 & 58.0 & 57.6 & \\
Hospital region (\%) & & & & \\
Northeast & 17.3 & 17.3 & 21.6 &
\end{tabular}




\begin{tabular}{|c|c|c|c|c|}
\hline Covariates & ESRD with LGIB (\%) & ESRD with LGIB (\%) & ESRD without LGIB (\%) & $p$-value \\
\hline South & 41.6 & 41.6 & 41.6 & \\
\hline West & 19.5 & 19.5 & 19.5 & \\
\hline \multicolumn{5}{|l|}{ Discharge } \\
\hline Routine & 40.5 & 40.5 & 40.1 & 0.23 \\
\hline Skill Nursing Facility & 2.8 & 2.8 & 2.7 & \\
\hline \multicolumn{5}{|c|}{ Charlson comorbidity Index (\%) } \\
\hline 0 or 1 & 0.1 & 0.1 & 0.1 & \\
\hline 2 & 9.2 & 9.2 & 9.2 & 1.00 \\
\hline 3 & 90.7 & 90.7 & 90.7 & \\
\hline \multicolumn{5}{|l|}{ Insurance Type (\%) } \\
\hline Medicare $(\%)$ & 77.2 & 77.2 & 77.0 & \\
\hline Medicaid (\%) & 9 & 9 & 9.2 & 0.65 \\
\hline Private $(\%)$ & 10.8 & 10.8 & 10.8 & \\
\hline Uninsured (\%) & 1.0 & 1.0 & 0.9 & \\
\hline Teaching Hospital (\%) & 75.1 & 75.1 & 75.0 & 0.89 \\
\hline \multicolumn{5}{|l|}{ Chronic Co-morbidity } \\
\hline $\mathrm{DM}(\%)$ & 56.3 & 56.3 & 56.3 & 1.00 \\
\hline HTN (\%) & 1.5 & 1.5 & 1.5 & 0.99 \\
\hline Obesity (\%) & 15.2 & 15.2 & 15.2 & 1.00 \\
\hline Dyslipidemia (\%) & 40.1 & 40.1 & 40.2 & 0.91 \\
\hline CAD (\%) & 38.5 & 38.5 & 37.9 & 0.65 \\
\hline CHF (\%) & 36.2 & 36.2 & 36.00 & 0.89 \\
\hline PVD (\%) & 9.5 & 9.5 & 9.5 & 0.99 \\
\hline Tobacco Smoking (\%) & 23.2 & 23.2 & 23.0 & 0.80 \\
\hline Alcohol Use (\%) & 13.1 & 13.1 & 12.9 & 0.60 \\
\hline Blood Thinners (\%) & 12.5 & 12.5 & 12.5 & 0.99 \\
\hline Chronic Lung disease (\%) & 10.3 & 10.3 & 10.2 & 0.89 \\
\hline
\end{tabular}

${ }^{*}$ denotes statistically significantly different result.

$\mathrm{DM}$, diabetes mellitus, HTN, hypertension, CAD, coronary artery disease, CHF, congestive heart failure

Table 3. Comparison of Primary and Secondary Outcomes: ERSD with LGIB vs. ESRD only

\begin{tabular}{llll}
\hline & Proportion of Outcomes (\%) & Proportion of Outcomes (\%) & Before Propensity Score Matchin \\
\hline Variable & ESRD with LGIB (\%) & ESRD without LGIB (\%) & Odds ratio \\
In-hospital Mortality & 7.9 & 4.5 & 1.3 \\
Sepsis & 13.9 & 8.7 & 1.4 \\
Acute Respiratory failure & 5.9 & 3.0 & 1.6 \\
Mechanical Ventilation & 10.2 & 4.5 & 1.8 \\
Pressor Requirements & 2.5 & 0.9 & 1.9 \\
Acute coronary syndrome & 4.8 & 2.7 & 1.6 \\
DIC & 1.2 & 0.8 & 2.9 \\
\hline
\end{tabular}

* denotes statistically significantly different result. DIC, disseminated intravascular coagulation

Table 4: Predictors of Mortality in ESRD with LGIB 


\begin{tabular}{llll}
\hline Variable & Odds Ratio & $P$-value & $95 \%$ CI \\
\hline Age & 1.02 & $0.00^{*}$ & $1.02-1.03$ \\
Anemia & 1.04 & $0.006^{*}$ & $1.59-1.91$ \\
Acute Coronary Syndrome & 1.8 & $0.00^{*}$ & $1.6-2.1$ \\
Acute Respiratory Failure & 1.29 & $0.00^{*}$ & $2.00-2.60$ \\
Mechanical Ventilation & 1.9 & $0.00^{*}$ & $3.5-4.4$ \\
Sepsis & 1.5 & $0.00^{*}$ & $4.1-5.08$ \\
\hline
\end{tabular}

*denotes statistically significantly different result

Table 5. Analysis for Length of Stay and Cost of Care

\begin{tabular}{llll}
\hline & ESRD with LGIB (\%) & ESRD without LGIB (\%) & $P$-value \\
\hline Mean LOS (Days) & $10.8 \pm 14.9$ & $6.3 \pm 8.5$ & $0.00^{*}$ \\
Mean Total Charge $(\$)$ & 37054 & 18080 & $0.00^{*}$ \\
\hline
\end{tabular}

*denotes statistically significantly different result

References:

1. Patel SD, Desai R, Patel U, et al. Thirty-Day Readmissions After Upper and Lower Gastrointestinal Hemorrhage: A National Perspective in the United States. J Clin Gastroenterol 2019;53 (8):582-90 doi: 10.1097/MCG.000000000001020[published Online First: Epub Date]|.

2. Lanas A, Garcia-Rodriguez LA, Polo-Tomas M, et al. Time trends and impact of upper and lower gastrointestinal bleeding and perforation in clinical practice. Am J Gastroenterol 2009;104 (7):1633-41 doi: 10.1038/ajg.2009.164[published Online First: Epub Date]|.

3. Whelan CT, Chen C, Kaboli P, Siddique J, Prochaska M, Meltzer DO. Upper versus lower gastrointestinal bleeding: a direct comparison of clinical presentation, outcomes, and resource utilization. J Hosp Med 2010;5 (3):141-7 doi: 10.1002/jhm.606[published Online First: Epub Date]|.

4. Peery AF, Crockett SD, Barritt AS, et al. Burden of Gastrointestinal, Liver, and Pancreatic Diseases in the United States. Gastroenterology 2015;149 (7):1731-41.e3 doi: 10.1053/j.gastro.2015.08.045[published Online First: Epub Date]|.

5. Trivedi H, Yang J, Szabo A. Gastrointestinal bleeding in patients on long-term dialysis. J Nephrol 2015;28 (2):235-43 doi: 10.1007/s40620-014-0132-6[published Online First: Epub Date]|.

6. Hagendorn R, Farkas N, Vincze A, et al. Chronic kidney disease severely deteriorates the outcome of gastrointestinal bleeding: A meta-analysis. World J Gastroenterol 2017;23 (47):8415-25 doi: 10.3748/wjg.v23.i47.8415[published Online First: Epub Date]|.

7. Ishigami J, Grams ME, Naik RP, Coresh J, Matsushita K. Chronic Kidney Disease and Risk for Gastrointestinal Bleeding in the Community: The Atherosclerosis Risk in Communities (ARIC) Study. Clin J Am Soc Nephrol 2016;11 (10):1735-43 doi: 10.2215/CJN.02170216[published Online First: Epub Date]|.

8. Luo JC, Leu HB, Huang $\mathrm{KW}$, et al. incidence of bleeding from gastroduodenal ulcers in patients with end-stage renal disease receiving hemodialysis. CMAJ 2011;183 (18):E1345-51 doi: 10.1503/cmaj.110299[published Online First: Epub Date]|.

9. Luo JC, Leu HB, Hou MC, et al. Nonpeptic ulcer, nonvariceal gastrointestinal bleeding in hemodialysis patients. Am J Med 2013;126 (3):264.e25-32 doi: 10.1016/j.amjmed.2012.09.010[published Online First: Epub Date]|. 
10. Toke AB. G.I. bleeding risk in patients undergoing dialysis. Gastrointest Endosc. United States, 2010:50-2.

11. Saeed F, Agrawal N, Greenberg E, Holley JL. Lower gastrointestinal bleeding in chronic hemodialysis patients. Int J Nephrol 2011;2011 :272535 doi: 10.4061/2011/272535[published Online First: Epub Date]|.

12. Kringen MK, Narum S, Lygren I, et al. Reduced platelet function and role of drugs in acute gastrointestinal bleeding. Basic Clin Pharmacol Toxicol 2011;108 (3):194-201 doi: 10.1111/j.17427843.2010.00643.x[published Online First: Epub Date]|.

13. Wasse H, Gillen DL, Ball AM, et al. Risk factors for upper gastrointestinal bleeding among end-stage renal disease patients. Kidney Int 2003;64 (4):1455-61 doi: 10.1046/j.1523-1755.2003.00225.x[published Online First: Epub Date]|.

14. Sood P, Kumar G, Nanchal R, et al. Chronic kidney disease and end-stage renal disease predict higher risk of mortality in patients with primary upper gastrointestinal bleeding. Am J Nephrol 2012;35 (3):216-24 doi: 10.1159/000336107[published Online First: Epub Date]|.

15. Kuo CC, Kuo HW, Lee IM, Lee CT, Yang CY. The risk of upper gastrointestinal bleeding in patients treated with hemodialysis: a population-based cohort study. BMC Nephrol 2013;14 :15 doi: 10.1186/14712369-14-15[published Online First: Epub Date]|.

16. Healthcare Cost and Utilization Project. . 2017.

17. Khera R, Angraal S, Couch T, et al. Adherence to Methodological Standards in Research Using the National Inpatient Sample. JAMA 2017;318 (20):2011-18 doi: 10.1001/jama.2017.17653[published Online First: Epub Date]|.

18. D'Agostino RB. Propensity scores in cardiovascular research. Circulation 2007;115 (17):2340-3 doi: 10.1161/CIRCULATIONAHA.105.594952[published Online First: Epub Date]|.

19. Austin PC.

20. Ohmori T, Konishi H, Nakamura S, Shiratori K. Abnormalities of the small intestine detected by capsule endoscopy in hemodialysis patients. Intern Med 2012;51 (12):1455-60 doi: 10.2169/internalmedicine.51.7190[published Online First: Epub Date]|.

21. Kalman RS, Pedrosa MC. Evidence-based review of gastrointestinal bleeding in the chronic kidney disease patient. Semin Dial 2015;28 (1):68-74 doi: 10.1111/sdi.12301[published Online First: Epub Date]|.

22. Freeman WJ, Weiss AJ, Heslin KC . Overview of U.S. Hospital Stays in 2016: Variation by Geographic Region. In: (HCUP) HCaUP, ed., 2018.

23. Gilbert J. Hospital readmission and length of stay. The Incidental Economist 2015.

24. Agency for Healthcare Research and Quality. Interim Update on 2013 Annual Hospital-Acquired Condition Rate and Estimates of Cost Savings and Deaths Averted From 2010 to 2013, 2015.

25. Healthcatalyst T. Patient-Centered LOS Reduction Initiative Improves Outcomes, Saves Costs. Cost Savings , Infection Reduction , Length of Stay , Patient Safety , Quality \& Process Improvements and Readmissions . 2016.

26. Garlo K, Williams D, Lucas L, et al. Severity of Anemia Predicts Hospital Length of Stay but Not Readmission in Patients with Chronic Kidney Disease: A Retrospective Cohort Study. Medicine (Baltimore) 2015;94 (25):e964 doi: 10.1097/md.0000000000000964[published Online First: Epub Date]|.

27. Daratha KB, Short RA, Corbett CF, et al. Risks of subsequent hospitalization and death in patients with kidney disease. Clin J Am Soc Nephrol 2012;7 (3):409-16 doi: 10.2215/CJN.05070511[published Online First: Epub Date]|. 
28. Weng SC, Shu KH, Tarng DC, et al. In-hospital mortality risk estimation in patients with acute nonvariceal upper gastrointestinal bleeding undergoing hemodialysis: a retrospective cohort study. Ren Fail 2013;35 (2):243-8 doi: 10.3109/0886022x.2012.747140[published Online First: Epub Date]|.

29. Yang JY, Lee TC, Montez-Rath ME, et al. Trends in acute nonvariceal upper gastrointestinal bleeding in dialysis patients. J Am Soc Nephrol 2012;23 (3):495-506 doi: 10.1681/asn.2011070658[published Online First: Epub Date]|.

30. Strate LL, Ayanian JZ, Kotler G, Syngal S. Risk factors for mortality in lower intestinal bleeding. Clin Gastroenterol Hepatol 2008;6 (9):1004-10; quiz 955- doi: 10.1016/j.cgh.2008.03.021[published Online First: Epub Date]|. 